\title{
CORRESPONDENCE
}

\section{Comments on the Recent Discussion Concerning the Article "Diathermal Heat Transport in a Global Ocean Model"}

\author{
ÁlVARO VIÚDEZ \\ Institute of Marine Sciences, CSIC, Barcelona, Spain
}

(Manuscript received 12 September 2019, in final form 23 October 2019)

\begin{abstract}
A 2019 comment by Hochet and Tailleux and the corresponding reply by Holmes et al. discuss the volume and mass balance on a control volume bounded by a given isotherm and the ocean free surface. This note partly reconciles the terms of discrepancy on volume or mass transport appearing in these publications by proving that the integral expressions in the comment of Hochet and Tailleux, using a particular parameterization of the moving surfaces in Cartesian coordinates, correspond to the mass transport across the moving surfaces, as long as the mass density is included, as given in direct vector notation by Holmes et al. in their reply.
\end{abstract}

\section{Introduction}

Two recent notes on the article by Holmes et al. (2019a, hereinafter HZE), namely, the comment by Hochet and Tailleux (2019, hereinafter HT) and the reply by Holmes et al. (2019b, hereinafter HZEr), discuss the volume and mass balance on a control volume bounded by a given isotherm and the ocean free surface. The main purpose of HT was to provide explicit mathematical expressions linking the volume change of HZE to the diabatic sources and sinks of heat. HZE correctly point out that the control volume is neither steady nor material and express the volume balance in terms of the volume flux across the appropriate moving boundary surfaces in direct vector notation [Eq. (3) in HZE complemented with the usual definition of volume flux across a moving surface given in Eq. (1) in HZEr]. On the other hand HT express the mass balance in terms of horizontal area integrals in Cartesian coordinates involving the fluid flow and the geometry of the pertinent surfaces [Eq. (15) in HT]. Since the relation between both mathematical expressions is not given, the reader may remain confused about which approach is in fact correct. This note reconciles both approaches by showing that, as long as the volume flux in HZEr is used

Corresponding author: Álvaro Viúdez, aviudez@cmima.csic.es instead of the mass flux, both mathematical expressions are exactly equal.

\section{Mass transport across moving surfaces}

For managing balance equations in integral form in arbitrary control volumes (i.e., not necessarily material or steady), it is useful to consider the kinematic identity (see Viúdez 2000) expressing the rate of change of mass in an arbitrary volume $\mathscr{T}$,

$$
\frac{d_{w}}{d t} \int_{\mathscr{V}} f d V=\frac{d}{d t} \int_{\mathscr{V}} f d V-\oint_{\partial \mathscr{C}} f(\mathbf{u}-\mathbf{w}) \cdot \mathbf{d} \mathbf{S}
$$

The above expression means that the rate of change of the extensive quantity with spatial density $f(\mathbf{x}, t)$ in an arbitrary volume $\mathscr{T}$, which is material with respect to the velocity $\mathbf{w}(\mathbf{x}, t)$, is equal to the rate of change of the extensive quantity in the fluid particles moving with velocity $\mathbf{u}(\mathbf{x}, t)$ located in the arbitrary volume $\mathscr{V}$ minus the flux or transport of $f$ relative to the moving boundary $\partial \mathscr{V}$. In the particular case $f=1$ Eq. (1) represents the volume balance, and in the case that $f$ is equal to the mass density $f(\mathbf{x}, t)=\rho(\mathbf{x}, t)$ Eq. (1) represents the mass balance

$$
\frac{d_{w}}{d t} \int_{\mathscr{V}} \rho d V=-\oint_{\partial \mathscr{V}} \rho(\mathbf{u}-\mathbf{w}) \cdot \mathbf{d} \mathbf{S}
$$


where we have assumed mass conservation $\rho+\rho \nabla \cdot \mathbf{u}=0$. The term in the right-hand side of Eq. (2) is equivalent to the term defined by HZEr [their Eq. (1)] when mass transport is considered instead of volume transport. We prove now that this balance is equal to the first equality in HT [their Eq. (15)], which for clarity is reproduced here as

$$
\frac{\partial M}{\partial t}=\iint_{A_{\theta}}\left(\rho_{s} \frac{\partial \eta}{\partial t}+\rho_{b} \frac{\partial h}{\partial t}\right) d x d y-\oint_{\partial \mathscr{C}} \rho \mathbf{u} \cdot \mathbf{n} d S .
$$

Since the term involving the fluid velocity $\mathbf{u}$ is already present in both Eqs. (2) and (3), it only remains to prove that

$$
\iint_{A_{\theta}}\left(\rho_{s} \frac{\partial \eta}{\partial t}+\rho_{b} \frac{\partial h}{\partial t}\right) d x d y \stackrel{?}{=} \oint_{\partial \mathscr{T}} \rho \mathbf{w} \cdot \mathbf{d S} .
$$

For the upper free surface $z=\eta(x, y, t)$, we define the function $s(x, y, z, t) \equiv z-\eta(x, y, t)$ so that the implicit equation for the free surface is $s(x, y, z, t)=0$. The velocity of displacement $\mathbf{w}_{s}(x, y, t)$ of this free surface satisfies the equation

$$
\frac{\partial s}{\partial t}+\mathbf{w}_{s} \cdot \nabla s=0
$$

and therefore

$$
\mathbf{w}_{s}(x, y, t) \equiv-\frac{\partial s}{\partial t} \frac{\nabla s}{|\nabla s|^{2}}=\frac{\partial \eta}{\partial t} \frac{\mathbf{n}_{s}}{\sqrt{1+\left|\nabla_{h} \eta\right|^{2}}},
$$

where the unit vector normal to the free surface

$$
\mathbf{n}_{s}(x, y, t) \equiv \frac{\nabla s}{|\nabla s|}=\frac{\mathbf{k}-\nabla_{h} \eta}{\sqrt{1+\left|\nabla_{h} \eta\right|^{2}}}
$$

is identical to that in HT [their Eq. (8)]. The differential area element parameterized by the Cartesian coordinates $(x, y)$ is

$$
\mathbf{d S}(x, y, t)=\sqrt{1+\left|\nabla_{h} \eta\right|^{2}} \mathbf{n}_{s} d x d y
$$

so that, at the upper surface, the right-hand side of Eq. (4) includes the term

$$
\rho \mathbf{w} \cdot \mathbf{d S}=\rho_{s} \frac{\partial \eta}{\partial t} d x d y .
$$

Note that, since $\mathbf{w}$ is the velocity of displacement of the integration surface, $\mathbf{w}$ and $\mathbf{d S}$ are tangent vectors.

In a similar way, for the lower, bottom surface, $z=-h(x, y, \theta, t)$, and for a constant temperature value $\theta$ we define the function $b(x, y, z, t) \equiv-z-h(x, y, \theta, t)$ so that the implicit equation for the isothermal surface $z=-h(x, y, \theta, t)$ is $b(x, y, z, t)=0$. Following the same steps carried out above with the free surface we obtain the velocity of displacement $\mathbf{w}_{b}(x, y, t)$ of the isothermal surface:

$$
\mathbf{w}_{b}(x, y, t) \equiv-\frac{\partial b}{\partial t} \frac{\nabla b}{|\nabla b|^{2}}=\frac{\partial h}{\partial t} \frac{\mathbf{n}_{b}}{\sqrt{1+\left|\nabla_{h} h\right|^{2}}},
$$

where the unit normal vector

$$
\mathbf{n}_{b}(x, y, t) \equiv \frac{\nabla b}{|\nabla b|}=-\frac{\mathbf{k}+\nabla_{h} h}{\sqrt{1+\left|\nabla_{h} h\right|^{2}}}
$$

is identical to that in HT [their Eq. (9)]. The differential area element at the bottom surface is

$$
\mathbf{d S}(x, y, t)=\sqrt{1+\left|\nabla_{h} h\right|^{2}} \mathbf{n}_{b} d x d y,
$$

and therefore at the bottom isothermal surface

$$
\rho \mathbf{w} \cdot \mathbf{d S}=\rho_{b} \frac{\partial h}{\partial t} d x d y .
$$

The relations in Eqs. (9) and (13) immediately prove Eq. (4) and hence confirm the equivalence of the formulations given in HZE, HZEr, and HT.

Using the above relations for the speed of displacement $\mathbf{w}$ of a moving surface defined by a relation of the form $F(x, y, z, t) \equiv z-f(x, y, t)=0$, and for a differential area element $\mathbf{d S}$ on this surface, it follows that

$$
\rho(\mathbf{u}-\mathbf{w}) \cdot \mathbf{d} \mathbf{S}=\rho_{f}\left(\frac{\partial f}{\partial t}+\mathbf{u}_{f h} \cdot \nabla_{h} f+w_{f}\right) d x d y,
$$

where $\mathbf{u}[x, y, f(x, y, t)]=\mathbf{u}_{f}(x, y, t)=\mathbf{u}_{f h}+w_{f} \mathbf{k}$ is the fluid velocity, and $\rho[x, y, f(x, y, t)]=\rho_{f}(x, y, t)$ is the mass density, on the surface $z=f(x, y, t)$. Thus, the term on the right-hand side of Eq. (14) may be kinematically interpreted as the differential flux of mass across the moving surface $z=f(x, y, t)$.

The general theory of mass and volume transport across isosurfaces of a balanced fluid property, that is, a fluid property obeying a balance equation, may be used to derive other balance configurations in the ocean, such as that in Walin (1982) for the heat balance or that in Garrett et al. (1995) for the average buoyancy balance (see Viúdez 2000, his appendix B).

\section{Conclusions}

In this note we have partly reconciled the terms of discrepancy on volume or mass transport appearing in 
two recent notes by proving that the integral expressions in Hochet and Tailleux (2019), using a particular parameterization of the moving surfaces in Cartesian coordinates, correspond to the mass transport across the moving surfaces, as long as the mass density is included, as given in direct vector notation by Holmes et al. (2019b).

Acknowledgments. I thank one anonymous reviewer for his/her comments. Partial support for this study was obtained through project RTI2018-100844-B-C33 (Spanish Ministry of Science, Innovation and Universities).

\section{REFERENCES}

Garrett, C., K. Speer, and E. Tragou, 1995: The relationship between water mass formation and the surface buoyancy flux, with application to Phillips' Red Sea Model. J. Phys. Oceanogr., 25, 1696-1705, https://doi.org/10.1175/15200485(1995)025<1696:TRBWMF>2.0.CO;2.

Hochet, A., and R. Tailleux, 2019: Comments on "Diathermal heat transport in a global ocean model." J. Phys. Oceanogr., 49, 2189-2193, https://doi.org/10.1175/JPO-D-19-0055.1.

Holmes, R. M., J. D. Zika, and M. H. England, 2019a: Diathermal heat transport in a global ocean model. J. Phys. Oceanogr., 49, 141-161, https://doi.org/10.1175/JPO-D-18-0098.1.

$\longrightarrow, \ldots$, and $-2019 \mathrm{~b}$ : Reply to "Comments on 'Diathermal heat transport in a global ocean model.", J. Phys. Oceanogr., 49, 2195-2197, https://doi.org/10.1175/JPO-D-19-0139.1.

Viúdez, A., 2000: Volume and mass transport across isosurfaces of a balanced fluid property. J. Phys. Oceanogr., 30, 1478-1485, https:// doi.org/10.1175/1520-0485(2000)030<1478:VAMTAI >2.0.CO;2.

Walin, G., 1982: On the relation between sea-surface heat flow and thermal circulation in the ocean. Tellus, 34, 187-195, https:// doi.org/10.3402/tellusa.v34i2.10801. 